\title{
Cardiac resynchronization therapy: pre and post pacemaker implantation issues
}

\section{Ashley Chin and Andrzej Okreglicki}

Cardiac Clinic, Groote Schuur Hospital, Cape Town, South Africa

\author{
Corresponding author: \\ Ashley Chin \\ Cardiac Clinic \\ Groote Schuur Hospital \\ Anzio Road \\ Observatory \\ 7925 \\ South Africa
}

Email:

ashley.chin@uct.ac.za

\section{ABSTRACT Cardiac resynchronization therapy (CRT)} has been shown to improve clinical outcomes in patients with systolic heart failure. However, up to one-third of patients do not benefit from CRT. Selection of appropriate patients with attention to heart failure management and optimization of pacemaker settings post-implantation will ensure the best chance of a favorable response to CRT.This article will review the current indications for CRT and provide recommendations regarding optimization and follow-up of patients with CRT. SAHeart 2008; 5:156-159

\section{INTRODUCTION}

Cardiac resynchronization therapy (CRT) is an effective treatment option for selected patients with severe advanced systolic heart failure.

This article will focus on practical management issues pre and post pacemaker insertion. Practical issues related to the implantation procedure have been reviewed elsewhere.(I)

\section{PRE-IMPLANTATION ISSUES}

Recent guidelines for selecting patients for CRT have been published. ${ }^{(2,3)}$
CRT is a class IA indication if the following criteria are fulfilled:

Severe symptomatic heart failure (NYHA III or ambulatory NYHA IV symptoms) despite optimal medical therapy

Depressed LV systolic function (LVEF $\leq 35 \%)$

Sinus rhythm with QRS duration $\geq 120 \mathrm{~ms}$

This recommendation is based on the results of 8 randomized clinical trials comprising 4017 patients on CRT.(4) All 8 trials have confirmed that CRT improves symptoms, exercise capacity and quality of life. Some trials have shown that CRT causes reverse left ventricular (LV) remodeling as shown by improvement in echocardiographic parameters (LV ejection fraction, LV size and mitral regurgitation). Two trials have shown that CRT reduces hospitalizations for heart failure and one trial showed CRT improves survival compared to medical therapy alone.

Each criterion warrants further clarification.

Patients should have severe symptoms (NYHA III, IV) secondary to heart failure, usually as a result of ischemic or dilated cardiomyopathy, despite optimal medical treatment before being considered for CRT. A common difficulty is distinguishing NYHA II from NYHA III symptoms in a patient. As the NYHA classification is extremely subjective, a better objective measurement of effort intolerance is the 6-minute walk test. Patients must cover the maximal distance possible in 6 minutes by walking and/or running and are allowed to rest if required. At Groote Schuur Hospital, we use a total distance $\geq 420 \mathrm{~m}$ to correlate with NYHA II. This objective measurement helps to classify symptoms while providing a reference for future comparison. CRT for patients with NYHA II symptoms and for patients with end-stage heart failure (bailout therapy) is under investigation and is currently not recommended.

Patients should be evaluated for CRT when all reversible and precipitating causes of heart failure have been addressed. It may take a further few months to titrate heart failure therapy to target doses recommended in clinical trials or to maximum doses tolerable by the patient. All patients should receive an ACE-I and/or ARB, beta-blocker and an aldosterone receptor blocker for prognostic and symptomatic benefit (unless contraindicated). Diuretics should be given for fluid retention and digoxin for additional symptomatic benefit. It is not uncommon for a patient to meet all the criteria for CRT only to improve to a NYHA I or II status once lifestyle and precipitating factors have been addressed and stabilized on optimal medical therapy. 
The principle of CRT is to diminish mechanical LV dyssynchrony to increase stroke volume. A QRS duration $\geq 120 \mathrm{~ms}$ is used as a surrogate marker of mechanical dyssynchrony, but is actually a measure of electrical dyssynchrony. Electrical dyssynchrony is not always accompanied by mechanical dyssynchrony. The detection of mechanical dyssynchrony by echocardiography (see below) in addition to electrical dyssynchrony (QRS duration $\geq 120 \mathrm{~ms}$ ) has been shown to improve the sensitivity and specificity of predicting response to CRT in some but not all trials. ${ }^{(4)}$ The definition and degree of mechanical dyssynchrony required to predict a favourable response to CRT stills needs to be fully investigated in clinical trials. ${ }^{(5)}$ Hence, the presence of mechanical LV dyssynchrony is not an essential criterion at present. Nevertheless, in resource-limited environments, echocardiographic measures of mechanical dyssynchrony should form part of the routine workup of a patient considered for CRT to best identify suitable candidates.

The most validated echocardiographic measures of mechanical intraventricular dyssynchrony are:

- M-Mode of LV in parasternal short-axis view (at papillary muscle level): Septal-to-posterior LV wall motion delay $\geq 130 \mathrm{~ms}^{(6)}$

- Colour-coded TDI (time to peak systolic velocities) of basal-septal and lateral segments of LV in 4 chamber view: Septal-to-lateral LV delay $\geq 60 \mathrm{~ms}^{(7)}$

Mechanical dyssynchrony is similarly not always accompanied by electrical dyssynchrony. A recent trial has shown that patients with echocardiographic evidence of mechanical dyssynchrony but without electrical dyssynchrony (QRS complexes $\leq 120 \mathrm{~ms}$ ) do not benefit from CRT. ${ }^{(8)}$

Most of the patients in trials had left bundle-branch block and were in sinus rhythm. It is uncertain at present whether patients with atrial fibrillation or right bundle-branch block benefit from CRT.(9,10) Studies show that patients with a combination of right bundle branch block and pulmonary hypertension do not benefit from CRT.

\section{POST-IMPLANTATION ISSUES}

Even when patients are selected for CRT on the basis of entry criteria used in trials, $20-30 \%$ of patients do not respond to CRT. Possible reasons for failure to respond include poor patient selection, implantation factors (e.g. poor lead placement) and post-implantation factors.

Patients should ideally be followed up at I month post implantation and then at 3-4 monthly intervals because of the higher complication rate compared to standard pacemakers. At each visit, patients should have a detailed clinical assessment, an ECG done and the pacemaker settings checked by a cardiologist and a medical technologist. The aim of each visit must include standard heart failure management and pacemaker interrogation. Pacemaker interrogation should consist of a standard pacemaker check-up (as for single or dual chamber pacemakers) and then specific CRT parameters should be checked and optimized.

\section{Clinical assessment and heart failure management}

Patients should be asked about improvement or deterioration in effort tolerance and repeated objective 6-minute walk tests performed. All randomized trials confirm a significant improvement in symptoms and exercise capacity with CRT. Mean NYHA function class improved by almost one class and the mean distance covered during a 6-minute walk test increased by a mean of $20 \%$ in most randomized trials. ${ }^{(3)}$ Most improvement in effort tolerance can be expected to occur in the first 6 months with sustained long-term benefit. Clinical examination of hemodynamic status may confirm responsiveness or detect response failure early before symptoms arise.

At each visit, the usual aggravating factors of heart failure should be sought for and treated. As heart failure is a progressive disease, patients should be told that compliance on all anti-heart failure medication is essential and that medication must be continued even if symptoms improve. Patients must be educated that CRT is complementary and not an alternative to good medical therapy. Adjustments to medications may be needed, especially a reduction in diuretics in those who have responded well to CRT.

\section{ECG}

The current ECG should be compared to previous ECGs as this should be the first clue of pacemaker malfunction or that both ventricles are not being paced (Figure I). A biventricular paced rhythm may show QRS complexes that are narrower than standard RV apical pacing, a change in axis and positive QRS complexes in VI-V3.

\section{Pacemaker check}

Pacemaker evaluation should follow the same systematic steps as for a single or dual-chamber pacemaker. It is inadequate just to check the pacing mode and settings and confirm that the battery is not depleted. Pacemaker interrogation includes:

Assessment of the underlying rhythm

Reviewing stored telemetry data for atrial and ventricular arrhythmias

Performing sensing tests of $P$ and $R$ waves

Testing atrial, LV and RV output thresholds. 
FIGURE I: ECGs in CRT: Both I2-lead ECGs are from the same patient with CRT

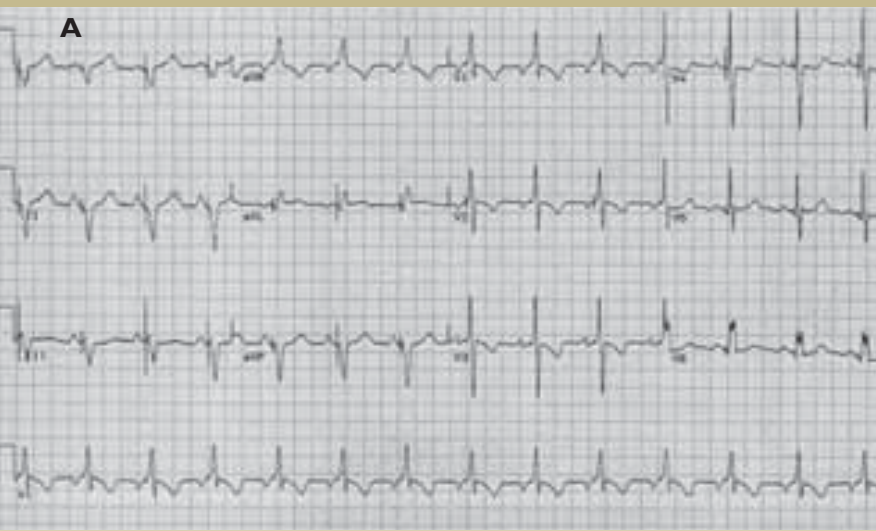

A: ECG was done after successful implantation of the biventricular $\mathrm{AV}$ sequential pacemaker. Note the QRS width and QRS axis (minus $105^{\circ}$ ).

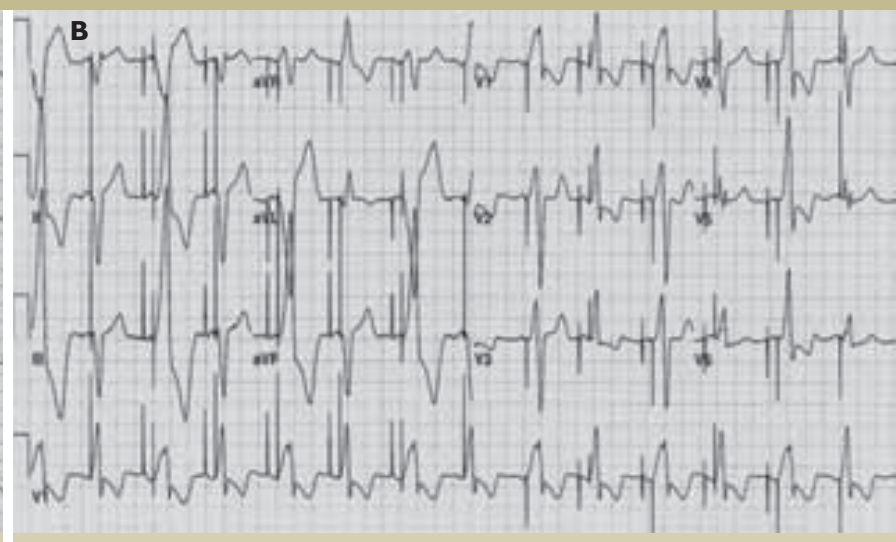

B: ECG was done at follow-up 3 months later. (Please note that the ECGs were done on different ECG recording machines and the relative sizes of the pacing spikes are artifactual and of no relevance.) This ECG shows biventricular pacing of only every second beat with morphology and axis identical to post-implantation. The alternate beats are much wider and have an axis of plus $105^{\circ}$. These beats have captured the left ventricle only. This was due to an increased right ventricular pacing threshold which happened to result in RV capture of only every second beat and failure for the other beats to capture, exposing the lone LV capture. This emphasizes the value of ECG in follow-up. This problem was easily corrected by pacemaker reprogramming with increased RV lead pacing amplitude.
LV thresholds are often higher than RV thresholds and doctors must ensure that an appropriate safety margin is set to ensure capture. Battery life and lead impedances should be documented at each visit to monitor for lead fracture and need for box replacement.

Interrogation of the pacemaker must confirm that the pacemaker is pacing both ventricles. This is unlike the usual backup demand pacemaker for most bradycardia indications where the percentage of total time paced may range from $<1-100 \%$ depending on the underlying rhythm. In CRT, pacing is not used as a back-up, but should be continuous and pace both ventricles. Without this, synchronization will not occur. Therefore interrogation should show that total ventricular pacing is near 100\%, a figure calculated by summing the total of atrial pacing/ventricular pacing plus atrial sensing/ventricular pacing (Figure 2). If atrial tachyarrhythmias (e.g. atrial fibrillation) are frequent, it is possible that the ventricle will be activated by normal conduction and not via the pacemaker which will result in bi-ventricular pacing not being near 100\% and CRT response will be suboptimal. The patient may then need an $\mathrm{AV}$ node ablation to maximize the ventricular pacing. If there are frequent ventricular ectopics, changing the lower ventricular rate setting to a higher rate may suppress ventricular ectopy.

Pacemakers must be set in DDD mode with the rate-response turned off, i.e. not DDDR mode unless there is chronotropic incompetence. This setting will ensure that patients are paced appropriate to their physiological requirements and will not lead to unnecessary ventricular pacing rates.
Synchronization involves not only synchronizing the left and right ventricles but also synchronizing the contraction of the atria and that of the ventricles. Current generation pacemakers enable separate programming of the delay between the contraction of the left and right ventricles $(\mathrm{V}-\mathrm{V}$ interval) and between the contraction of the atria and ventricles ( $A-V$ interval).

To achieve the best cardiac output, which after all is the main indication for CRT, the timing of atrial contraction before the occurrence of ventricular contraction is critical in order to take full advantage of the "atrial kick" and minimize pre-systolic mitral regurgitation and maximize stroke volume. Generally, a short A-V time needs to be programmed.

\section{FIGURE 2: Interrogation of CRT devices}

\section{Initial Interrogation Report}

Pacing (\% of total)

AS - VS $\quad<0.1 \%$

AS - VP $\quad 14.0 \%$

AP - VS $\quad 0.0 \%$

AP - VP $\quad 86.0 \%$

On interrogation of the biventricular pacemaker, the percentage of pacing must be noted. In this example from a Medtronic dual chamber biventricular pacemaker, ventricular pacing adds up to $100 \%$ of total ventricular beats. The target is $>90 \%$. Please note that this is what the pacemaker "believes" it is doing. Without additional assessment, it remains unknown whether, despite 100\% ventricular pacing, biventricular pacing is actually achieved. The patient in Figure I had almost 100\% ventricular pacing at follow-up pacemaker interrogation despite only achieving biventricular capture half the time.

Abbreviations: $A S=$ Atrial sensing, $V S=$ Ventricular sensing, $A P=$ Atrial pacing, $\mathrm{VP}=$ Ventricular pacing 
It is recommended that standard settings be used. At implantation, the standard A-V intervals programmed are: paced $110 \mathrm{~ms}$, sensed $100 \mathrm{~ms}$ with rate adaptive $A-V$ interval changes turned off. In most patients, this empiric setting is sufficient. In non-responders, however, more careful adjustment is appropriate to set the AV intervals to maximize stroke volume. This is a time-consuming process done with echocardiography by maximizing LV stroke volume (LV stroke volume = cross sectional area of LVOT $X$ velocity time integral) while testing a series of different AV intervals.

The other adjustment that may improve response is the interventricular pacing interval or $V-V$ time. At implantation, the standard $V-V$ interval is set at $\mathrm{Oms}$. In poor or non-responders, the $\mathrm{V}-\mathrm{V}$ interval can be adjusted to obtain maximal electrical synchrony between the 2 ventricles. This can be done by adjusting the $\mathrm{V}-\mathrm{V}$ interval (e.g. making the LV pacing earlier than the RV in 20ms intervals) while observing the width of the paced QRS complex. Theoretically, the narrowest paced QRS should correlate with the least electrical dyssynchrony. Although this is relatively simple to do and does not require echocardiography, this method of optimization has not been shown to improve clinical outcomes. Alternatively, stroke volume can be measured as described above while the $\mathrm{V}-\mathrm{V}$ intervals are adjusted to maximize aortic stroke volume. In studies, the highest stroke volume is usually obtained by pacing the LV first (usually $40 \mathrm{~ms}$ ). It must be re-iterated that the above adjustments are extremely labour intensive and time consuming with little proven clinical benefit. Standard settings are usually adequate unless the clinical response is poor. In these non-responders, echocardiographic optimization can be performed. (II) It is unclear whether A-V optimization should preceed $V-V$ optimization or vice versa. More recently, some pacemakers offer quick automated optimization using intracardiac electrograms from the atria, RV and LV to optimize $A-V$ and $V-V$ delays. Although this method has a close correlation with stroke volume derived from aortic VTI with echocardiography, clinical outcome data is lacking. ${ }^{(12)}$

\section{Echo follow-up}

Ideally all patients should have a repeat echocardiogram after implantation (usually at 3-6 months). Results of several trials indicate that CRT causes reverse LV remodeling, decreases LV end-systolic and end-diastolic volumes and increases LVEF. Randomized trials typically demonstrated a mean 15\% absolute reduction in LV end-diastolic diameter and up to a mean $6 \%$ increase in LVEF following CRT.(3) These effects were greater in patients with non-ischemic than in those with ischemic heart disease. The CARE-HF study confirmed that the reverse remodeling was sustained up to 18 months. ${ }^{(13)}$

\section{Patient advice}

Driving is usually permitted I week after device implantation. Patients should be counseled about electromagnetic interference as a potential cause of pacemaker malfunction. Sources of in-hospital electromagnetic interference include electrocautery, lithotripsy, radiofrequency ablation and magnetic resonance imaging. Out-of-hospital sources, including mobile telephones and electronic surveillance equipment, are much less of a problem.

\section{CONCLUSIONS}

CRT is an important treatment option in some patients with advanced systolic heart failure. Selection of appropriate patients with attention to heart failure management and optimization of pacemaker settings post-implantation will ensure the best chance of a favourable response to CRT.

\section{REFERENCES:}

I. Okreglicki A. Cardiac resynchronization therapy for heart failure: practical issues and tips. SA Heart 2004 ( I);43-51.

2. Epstein AE, DiMarco JP, Ellenbogen KA, et al. 2008 guidelines for device-based therapy of cardiac rhythm abnormalities: executive summary. J Am Coll Cardiol 2008;5। 2085-2105.

3. Vardas PE, Auricchio A, Blanc J, et al. Guidelines for cardiac pacing and cardiac resynchronization therapy. Eur Heart J 2007;28:2256-95.

4. Bax J), Abraham T, Barold SS, et al. Cardiac resynchronization therapy. J Am Coll Cardiol 2005;46:2153-67.

5. Chung ES, Leon AR, Tavazzi $L$, et al. Results of the Predictors of Response to CRT (PROSPECT) Trial. Circulation 2008; 1 17:2608-16.

6. Pitzalis MV, lacoviello M, Romito R, et al. Cardiac resynchronization therapy tailored by echocardiographic evaluation of ventricular asynchrony. J Am Coll Cardiol. 2002; 40:1615-22.

7. Bax JJ, Marwick TH, Molhoek SG, et al. Left ventricular dyssynchrony predicts benefit of cardiac resynchronization therapy in patients with end-stage heart failure before pacemaker implantation. Am J Cardiol. 2003;92: I 238-40.

8. Beshai JF, Grimm RA, Nagueh SF, et al. Cardiac resynchronization therapy in heart failure with narrow QRS complexes. N Engl J Med 2007;357:246I-7I.

9. Greenberg B, Mehra MR. Should all patients with heart failure and intraventricular conduction defect or dyssynchrony receive cardiac resynchronization therapy? Circulation 2006; | |4:2685-9|.

10. Abraham WT. Cardiac resynchronization therapy is important for all patients with congestive heart failure and ventricular dyssynchrony. Circulation 2006; | | 4:2692-98.

I I. Bax J), Abraham T, Barold SS, et al. Cardiac resynchronization therapy. J Am Coll Cardiol 2005;46:2168-82

12. Baker JH 2nd, McKenzie J 3rd, Beau S, et al. Acute evaluation of programmer-guided AV/PV and $\mathrm{V}$ delay optimization comparing an IEGM method and echocardiogram for cardiac resynchronization therapy in heart failure patients and dual-chamber ICD implants. J Cardiovasc Electrophysiol. 2007; | 8: | 85-9|.

13. Cleland J, Daubert JC, Erdmann E, et al. The effect of cardiac resynchronization on morbidity and mortality in heart failure. N Engl J Med 2005;352:1539-49. 